05.5

\title{
Неравновесные состояния в ВТСП-композитах второго поколения при сверхкритических импульсных токовых воздействиях
}

\author{
(ㄱ И.В. Анищенко, С.В. Покровский, М.А. Осипов, Д.А. Абин, Д.И. Грицаенко, И.А. Руднев \\ Национальный исследовательский ядерный университет „МИФИ“, Москва, Россия \\ ๑ E-mail: IARudnev@mephi.ru
}

Поступило в Редакцию 14 апреля 2021 г.

В окончательной редакции 22 июня 2021 г.

Принято к публикации 23 июня 2021 г.

\begin{abstract}
Представлены результаты исследования процессов переключения ВТСП-композитов из сверхпроводящего в резистивное состояние при микросекундных токовых импульсах. Применялось два режима импульсной токовой нагрузки: с амплитудой $\sim 1.1 I_{c}$ (так называемый „мягкий“ режим, $I_{c}-$ величина критического тока) и с амплитудой $\sim 3 I_{c}$ (,жесткий“ режим). Показана возможность пропускания сверхкритических токов через ленту без деградации характеристик сверхпроводника. Для анализа процессов, происходящих в ленте при протекании тока, была разработана 2D FEA (finite element analyses) модель, при помощи которой проведен расчет динамического сопротивления сверхпроводящего слоя ВТСП-композита и продемонстрированы процессы перераспределения тока между слоями ленты.
\end{abstract}

Ключевые слова: ВТСП-композиты, сверхпроводящий ключ, неравновесные состояния, устойчивое переключение, необратимое переключение.

DOI: 10.21883/PJTF.2021.19.51508.18828

В настоящее время высокотемпературные сверхпроводящие (ВТСП) композиты начинают заменять низкотемпературные сверхпроводники в коммутационных системах и часто становятся неотъемлемой частью таких устройств, как сверхпроводящие накопители энергии [1], ограничители тока [2], томографы МРТ [3]. Экспериментальные исследования и численное моделирование неравновесных состояний, возникающих в сверхпроводниках в условиях быстрой токовой сверхкритической (т.е. с амплитудой импульса, превышающей величину критического тока $I_{c}$ ) нагрузки, являются важной задачей, решение которой необходимо для проектирования, создания и оптимизации коммутационных устройств различного назначения.

В настоящей работе рассматриваются процессы переключения ВТСП-композита в режиме „жесткой“ токовой нагрузки $\left(\sim 3 I_{c}\right)$ и в режиме „мягкой“ токовой нагрузки $\left(\sim I_{c}\right)$. Экспериментальные исследования процессов переключения ВТСП-композитов при импульсных токовых нагрузках были выполнены с использованием коммерческих лент производства СуперОкс [4]. Использовались ВТСП-ленты шириной $4 \mathrm{~mm}$ с медным покрытием (толщина ВТСП-слоя $1 \mu \mathrm{m}$, толщина слоя серебра $2 \mu \mathrm{m}$, толщина медного покрытия $20 \mu \mathrm{m}$, толщина подложки $80 \mu \mathrm{m})$. Критический ток композита при температуре кипения жидкого азота составлял $I_{c}=150$ А. Эксперименты по импульсной токовой нагрузке проводились в диапазоне амплитуд импульсов 0-460 А с длительностью импульса $50-250 \mu \mathrm{s}$ и фронтом нарастания импульса $1.5-5 \mu \mathrm{s}$. Электрические измерения осуществлялись по четырехконтактной схеме с расстоянием между потенциальными контактами $6 \mathrm{~mm}$ в жидком азоте. На рис. 1 представлены зависимости тока и напряжения через образец в режимах мягкой $(a)$ и жесткой $(b)$ токовой нагрузки. В первом режиме токовой нагрузки амплитуда импульса и время нарастания фронта воздействия составили 160 А и $3 \mu \mathrm{s}$, во втором режиме - $460 \mathrm{~A}$ и $1.5 \mu \mathrm{s}$ соответственно. В режиме жесткой токовой нагрузки при увеличении амплитуды транспортного тока напряжение на образце продолжает неуклонно расти вплоть до полного снятия токовой нагрузки, тогда как в режиме мягкой токовой нагрузки наблюдается обратимое переключение ВТСП-композита, при котором при продолжении роста величины транспортного тока напряжение на образце начинает снижаться. Поведение сверхпроводящей ленты при пропускании импульса тока с временем роста порядка $1 \mu \mathrm{s}$ кардинально отличается от аналогичных результатов, полученных на импульсах с длительностями порядка миллисекунд [5,6], для которых зачастую характерны более стабильное поведение и задержка возникновения напряжения на образце во время приложения токового импульса.

Для объяснения процессов, происходящих в ВТСПленте при протекании токов, в несколько раз превышающих критический (жесткий режим), с помощью подхода, описанного в работе [7], была разработана двумерная модель для численного анализа таких систем на основе метода конечных элементов (finite element analyses, FEA). В отличие от работы [7] расчетный алгоритм был оптимизирован для расчета композитов с медным покрытием. Разработанная модель применима для оценки значений токов, протекающих в каждом слое ВТСП-ленты, и сопротивления сверхпроводящего слоя $\rho(J, T)$ в зависимости от приложенного тока и локальной температуры. В основе описания физики тепловых процессов заложено стандартное уравнение 

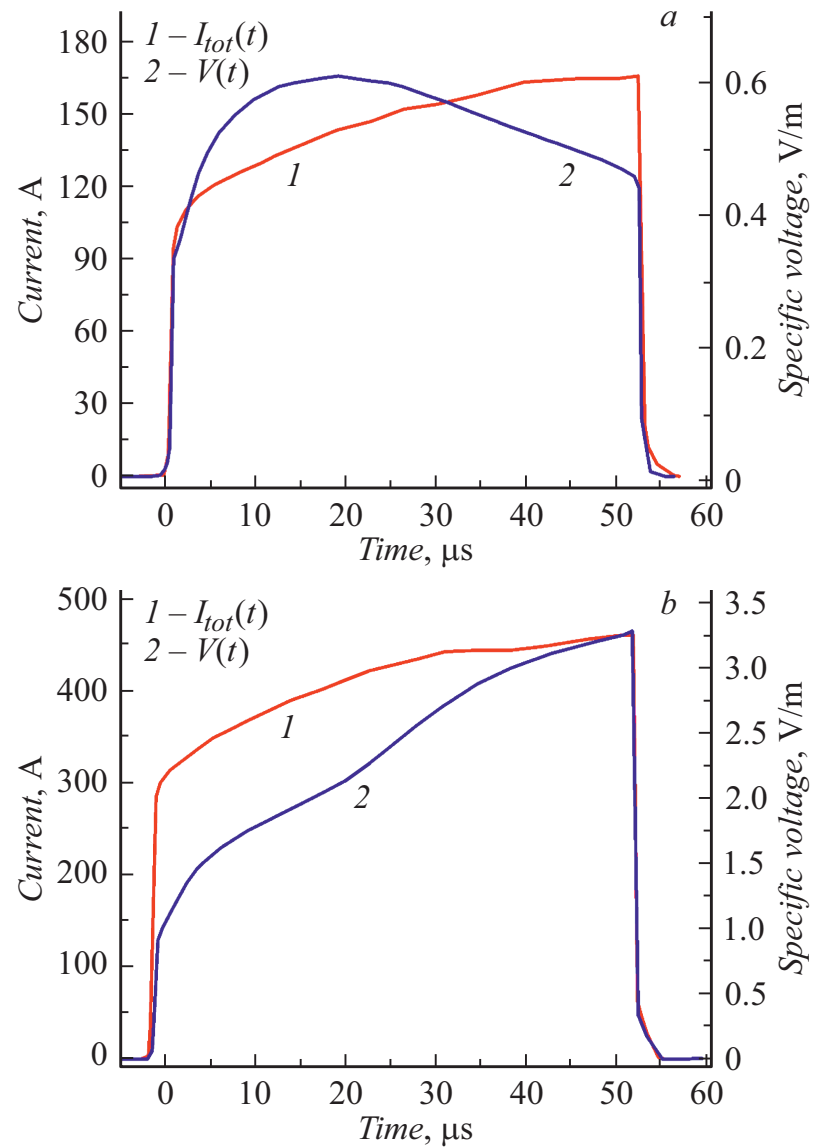

Рис. 1. Временнб́е зависимости тока и напряжения на единицу длины между потенциальными контактами на образце при воздействии токового импульса. $a-$ режим мягкой нагрузки (амплитуда импульса $160 \mathrm{~A}\left(\sim I_{c}\right)$, скорость ввода тока $50 \mathrm{~A} / \mu \mathrm{s}), b-$ режим жесткой нагрузки (амплитуда импульса $460 \mathrm{~A}\left(\sim 3 I_{c}\right)$, скорость ввода тока $\left.300 \mathrm{~A} / \mu \mathrm{s}\right)$.

теплопередачи в твердых телах. Локальное тепловыделение во всех слоях ВТСП-ленты рассчитывается как произведение плотности тока и напряженности электрического поля. Модель также учитывает температурные зависимости теплопроводностей, теплоемкостей, электросопротивлений и плотностей всех использованных материалов. В рамках модели реализован режим охлаждения ВТСП-композита жидким азотом. При этом предусмотрены многократная смена режимов кипения хладагента с конвективного на пузырьковое и обратно, дополнительный перегрев (задержка кипения), гистерезисный характер кривой кипения жидкого азота $[8,9]$.

В качестве источника тепла $Q$ выступают все слои ленты, $Q=J(t) E(t)$, где напряженность электрического поля $E(t)$ и плотность токов $J(t)$ определяются исходя из экспериментальных данных как

$$
\begin{aligned}
& E(t)=\frac{V_{\text {meas }}(t)}{l}, \\
& J(t)=\frac{E(t)}{\rho_{\text {mat }}(T)},
\end{aligned}
$$

где $V_{\text {meas }}(t)$ - зависимость экспериментально измеренного напряжения на расстоянии $l$ между потенциальными контактами от времени, $\rho_{\text {mat }}(T)-$ сопротивление материала, температурная зависимость которого точно известна для всех слоев ленты, кроме сверхпроводящего. Ток в каждом материале $I_{i}^{\text {mat }}$ определяется путем интегрирования плотности тока по площади поперечного сечения соответствующего слоя $S_{i}^{\text {mat }}$. С использованием экспериментально измеренного общего тока $I_{t o t}(t)$ через ВТСП-ленту ток через сверхпроводящий слой может быть найден как

$$
I_{\mathrm{HTS}}(T(t))=I_{\text {tot }}(t)-\sum_{i=1}^{n_{\text {mat }}} I_{i}^{\text {mat }}(T(t)) .
$$

И наконец, сопротивление ВТСП-слоя $\rho_{\text {HTS }}(J, T)$ определяется в соответствии с выражением

$$
\rho_{\mathrm{HTS}}(J, T)=\frac{V_{\text {meas }}(t)}{J_{\mathrm{HTS}}(T(t))} \frac{S_{\mathrm{HTS}}}{l} .
$$

Следует отметить, что в рамках данного модельного представления рост температуры при протекании тока через несверхпроводящие слои приводит к увеличению сопротивления этих слоев и снижению величины тока, протекающего через них. В таком случае ток через ВТСП-слой эффективно увеличится (см. уравнение (3)). Это приведет к тому, что сопротивление ВТСП-слоя, рассчитанное в соответствии с выражением (4), будет тем меньше, чем больше величина тока, протекающего через сверхпроводник. Этот эффект не вызывает значительной ошибки в тех случаях, когда основная часть токов протекает в сверхпроводящем слое, а толщина стабилизирующих слоев невелика, как это было в работе [10]. В нашем случае при наличии массивного медного слоя, способного переносить при криогенных температурах достаточно высокие токи, необходимо вводить поправочный коэффициент для сопротивления ВТСП-слоя. В связи с этим в рамках модели в области ВТСП-слоя вводится дополнительный объемный источник тепла, мощность тепловыделения в котором пропорциональна величине гистерезисных потерь в ВТСП-слое во время нарастания импульса и реальному сопротивлению слоя $[10,11]$. Описанный алгоритм был реализован с использованием модуля Heat Transfer in Solids программного пакета Comsol Multiphysics. Ввиду того что геометрия системы воспроизводит реальную архитектуру ВТСП-лент, при создании конечно-элементной сетки использованы специальные механизмы адаптации, такие как многомасштабное структурирование и протяжка сетки через тонкие слои ВТСП-ленты [12].

Проведем анализ процессов перераспределения токов между слоями ВТСП-ленты на примере более жесткого режима нагрузки, для которого неравновесные процессы наиболее выражены. На рис. 2 приведены зависимости тока во всех слоях ВТСП-ленты от времени в течение всего импульса $(a)$ и в течение нарастания токового 

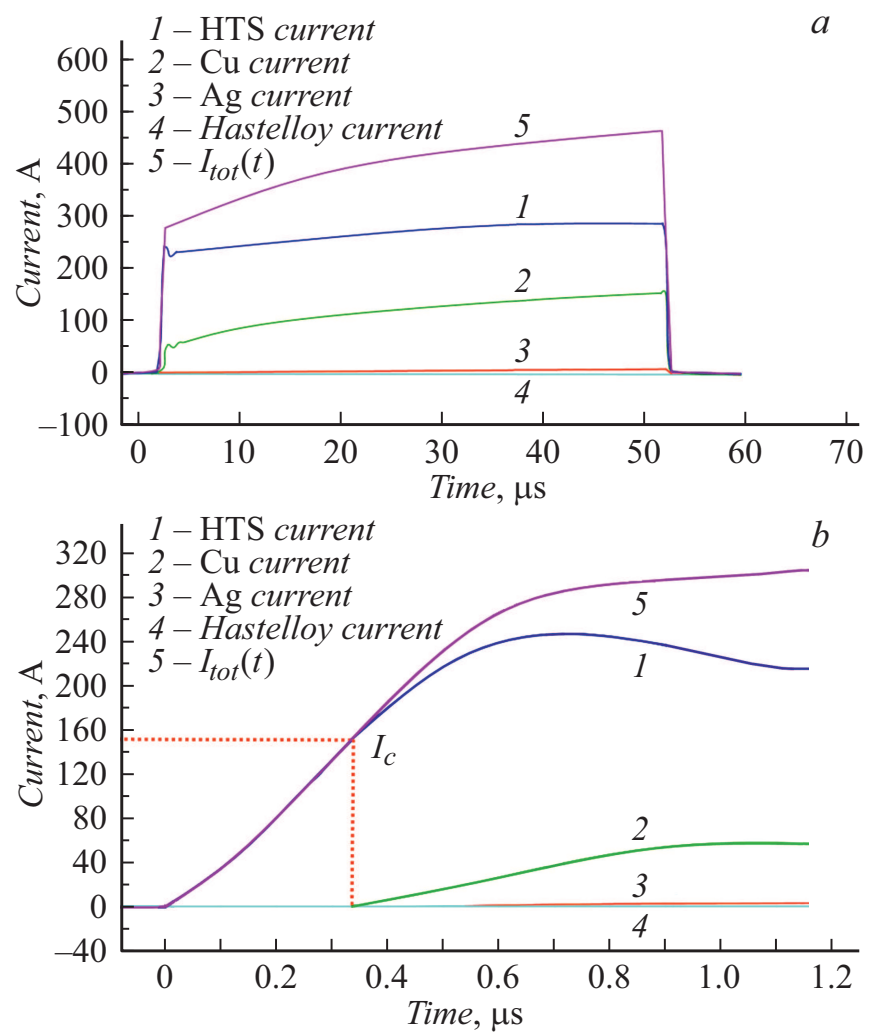

Рис. 2. Зависимости тока во всех слоях ВТСП-ленты от времени в течение всего импульса $(a)$ и в течение нарастания токового фронта $(b)$.

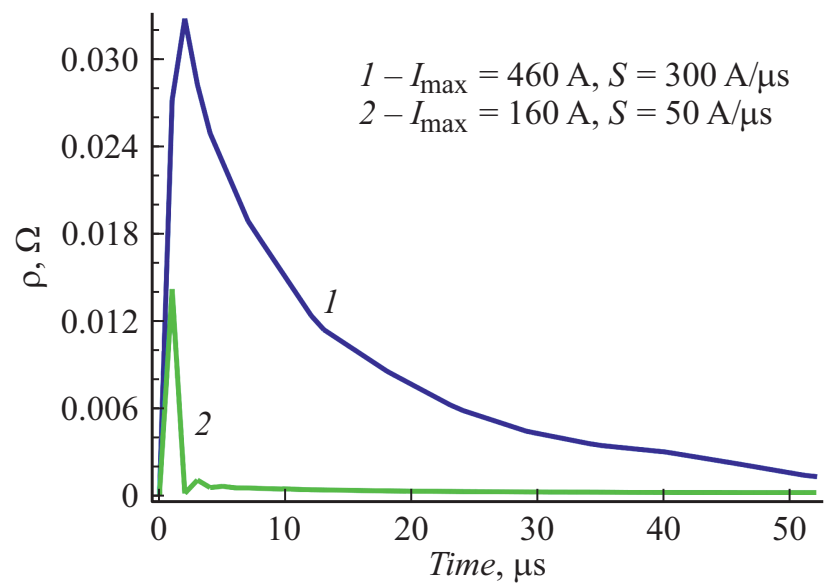

Рис. 3. Динамическое сопротивление ВТСП-слоя композитной ленты в жестком (амплитуда импульса $460 \mathrm{~A}\left(\sim 3 I_{c}\right)$, скорость ввода тока $300 \mathrm{~A} / \mu \mathrm{s})$ и мягком (амплитуда импульса $160 \mathrm{~A}\left(\sim I_{c}\right)$, скорость ввода тока $\left.50 \mathrm{~A} / \mu \mathrm{s}\right)$ режимах нагрузки. Длительность импульса $50 \mu \mathrm{s}$.

фронта $(b)$. Отметим, что на рис. $2, a$ в начале приложения импульса имеются ярко выраженные особенности, связанные с процессами перераспределения токов и установлением равновесного состояния в системе. При более детальном рассмотрении временно́го промежутка, связанного с нарастанием токового фронта (рис. 2, $b$ ), можно увидеть, что вплоть до того момента, пока величина тока не достигла значения критического тока ВТСП-ленты, весь ток протекает в сверхпроводящем слое. Затем ток появляется в медном слое, выполняющем стабилизирующую функцию. Слой серебра ввиду малой толщины переносит лишь очень малую часть токов, а слой подложки не участвует в процессах перераспределения токов между слоями ленты ввиду своей низкой проводимости. Кроме того, с использованием разработанного подхода возможно определение динамического сопротивления ВТСП-слоя ленты, которое может быть интегрировано в модели с электрической схемой для расчета параметров устройств, в цепи которых имеются сверхпроводящие элементы. Динамическое сопротивление ВТСП-слоя ленты в течение токового импульса длительности $50 \mu \mathrm{s}$ для жесткого и мягкого режимов нагрузки приведено на рис. 3. Отметим, что рассчитанное сопротивление не является общей характеристикой материала, а имеет место лишь при заданных условиях воздействия. Поэтому данное расчетное сопротивление ВТСП-слоя может быть использовано лишь для расчета устройств, работающих в аналогичных режимах нагрузки.

Таким образом, в работе продемонстрирована возможность пропускания через ВТСП-ленту импульсного тока c амплитудой, превышающей критический ток ленты в 3 раза, без деградации характеристик сверхпроводника. При этом наблюдается переключение в резистивное состояние, сохраняющееся вплоть до окончания действия импульса. Моделирование нестационарных процессов продемонстрировало динамику перераспределения токов между слоями. Показано, что с использованием разработанной модели возможно определение критического тока и динамического сопротивления ВТСП-слоя. Полученные данные могут быть использованы при конструировании быстродействующих переключающих устройств на основе высокотемпературных сверхпроводящих композитов. Отметим, что экспериментальные данные, представленные в настоящей работе, были получены с использованием ВТСП-лент с высокой степенью однородности критического тока. Расчетная модель также рассматривает случай полной однородности характеристик слоев лент и идеальный термический и электрический контакт между ними. Однако наличие межзеренных границ и температурной нестабильности магнитных потоков может существенно повлиять на процессы переключения в случае сильно неоднородных ВТСП-слоев [13]. Кроме того, отдельного внимания заслуживают вопросы стабилизации лент при импульсных токовых воздействиях и влияния процессов перераспределения тепла в слоях $[6,14]$. Экспериментальное и численное изучение этих вопросов является предметом последующих исследований. 


\section{Финансирование работы}

Исследование выполнено при финансовой поддержке Российского фонда фундаментальных исследований в рамках научного проекта № 17-29-10024, а также научного проекта № 20-38-90144 (И.В. Анищенко) по конкурсу „Аспиранты“.

\section{Конфликт интересов}

Авторы заявляют, что у них нет конфликта интересов.

\section{Список литературы}

[1] K. Sawa, M. Suzuki, M. Tomita, M. Murakami, IEEE Trans. Components Packaging Technol., 25, 415 (2002). DOI: $10.1109 /$ TCAPT.2002.804605

[2] D. Park, K. Chang, S. Yang, Y.J. Kim, M. Ahn, Y.-S. Yoon, H. Kim, J.-W. Park, T. Ko, IEEE Trans. Appl. Supercond., 19, 1896 (2009). DOI: 10.1109/TASC.2009.2018069

[3] S.B. Kim, M. Takahashi, R. Saito, Y.J. Park, M.W. Lee, Y.K. Oh, H.S. Ann, Phys. Procedia, 65, 149 (2015). DOI: 10.1016/j.phpro.2015.05.088

[4] S. Lee, V. Petrykin, A. Molodyk, S. Samoilenkov, A. Kaul, A. Vavilov, V. Vysotsky, S. Fetisov, Supercond. Sci. Technol., 27, 044022 (2014). DOI: 10.1088/0953-2048/27/4/044022

[5] I.V. Anischenko, S.V. Pokrovskii, I.A. Rudnev, J. Phys.: Conf. Ser., 1686, 012041 (2020). DOI: $10.1088 / 1742-6596 / 1686 / 1 / 012041$

[6] L. Antognazza, M. Decroux, M. Therasse, M. Abplanalp, IEEE Trans. Appl. Supercond., 21, 1213 (2011). DOI: 10.1109/TASC.2010.2100351

[7] N. Riva, S. Richard, F. Sirois, C. Lacroix, B. Dutoit, F. Grilli, IEEE Trans. Appl. Supercond., 29, 6601705 (2019). DOI: 10.1109/TASC.2019.2902038

[8] M.-H. Shi, J. Ma, B-X. Wang, Int. J. Heat Mass Transfer, 36, 4461 (1993). DOI: 10.1016/0017-9310(93)90130-X

[9] С.В. Самойленков, В.И. Щербаков, Д.Р. Кумаров, Д.А. Горбунова, Письма в ЖТФ, 46 (1), 28 (2020). DOI: $10.21883 /$ PJTF.2020.01.48860.18047

[10] В.В. Зубко, С.С. Фетисов, Кабели и провода, № 1 (369), 3 (2018). https://www.elibrary.ru/item.asp?id $=32581263$

[11] В.А. Мальгинов, Письма в ЖТФ, $45(22), 7$ (2019). DOI: $10.21883 /$ PJTF.2019.22.48640.17822

[12] V.M. Rodriguez-Zermeno, N. Mijatovic, C. Traeholt, T. Zirngibl, E. Seiler, A.B. Abrahamsen, N.F. Pedersen, M.P. Sorensen, IEEE Trans. Appl. Supercond., 21, 3273 (2011). DOI: 10.1109/TASC.2010.2091388

[13] A.V. Bobyl, D.V. Shantsev, T.H. Johansen, M. Baziljevich, Y.M. Galperin, M.E. Gaevski, Supercond. Sci. Technol., 13, 183 (2000). DOI: 10.1088/0953-2048/13/2/312

[14] F. Sirois, J. Coulombe, A. Bernier, IEEE Trans. Appl. Supercond., 19, 3585 (2009). DOI: 10.1109/TASC.2009.2018304 